\title{
Los afrodescendientes en los censos latinoamericanos, 1776-2011
}

George R. Andrews

University of Pittsburgh

Desde comienzos del siglo veintiuno, los censos de los países latinoamericanos han prestado creciente atención a la raza y la etnicidad, reuniendo información sobre afrodescendientes, indígenas y otras minorías étnicas. Este interés por cuantificar los grupos raciales y étnicos es un cambio radical en relación con las prácticas en la región durante el siglo XX, aunque no es una novedad en la historia de la región. Este ensayo considera la historia de larga duración del levantamiento de censos en América Latina y las formas en las que los individuos de ascendencia africana fueron contados en diferentes países y en diferentes momentos. Después de un breve análisis de los censos de los siglos XVIII, XIX y XX, examina la reciente ronda de censos nacionales realizados en 2010 y algunas de las formas en las que actualmente ha sido conceptualizada y cuantificada la negritud. ${ }^{2}$

\section{Contando la raza: una breve historia}

Los funcionarios coloniales españoles realizaron por primera vez censos de población en gran escala (de población indígena en Perú, y de la totalidad de la población en México) a fines del siglo XVII y en la primera mitad del XVIII. Pero los censos de la totalidad del imperio recién comenzaron en 1776. Como parte del esfuerzo de la monarquía borbónica por aumentar la recaudación impositiva y ampliar las milicias coloniales, la Corona ordenó recuentos exhaustivos de la población en todas las colonias, detallando el sexo, estado civil

\footnotetext{
${ }^{1}$ From Afro-Latin America: Black Lives, 16oo-2ooo by George R. Andrews. Copyright (c) 2016 by the President and Fellows of Harvard College. Used by permission. All rights reserved. Traducción del inglés: Lic. Ana María Rodríguez Ayçaguer. 2 Por una investigación más detallada de esta historia, ver LOVEMAN, Mara, National Colors: Racial Classification and the State in Latin America, New York, Oxford University Press, 2014.
} 
y la calidad de sus habitantes. Los funcionarios respondieron con una serie de censos realizados entre 1777 y 1810 . Reflejando las condiciones locales, dichos conteos variaron en su tratamiento de las castas y categorías raciales. La ausencia de población indígena en Cuba y Puerto Rico hizo que los funcionarios en dichas islas concentraran su atención en las distinciones raciales dentro de la población negra esclava y libre, registrando el número de negros y mulatos (individuos con ascendencia europea y africana) en cada una. ${ }^{3}$ En regiones del imperio con una gran población indígena, los funcionarios prestaron menor atención a la categoría racial de "mulato", en algunos casos prescindiendo totalmente de ella. En Perú, los censistas contaron a los mulatos como mestizos, negros libres o esclavos (quiere decir que los sumaron a las categorías de mestizos, negros libres, etc.). ${ }^{4}$ Los funcionarios del virreinato de Nueva Granada (actuales Colombia, Venezuela, Panamá y Ecuador) crearon la categoría "libres de todos los colores" que incluía mestizos, mulatos, zambos (individuos con ascendencia indígena y africana) y negros libres. En la mayor parte del virreinato, esta categoría resultó ser la mayoritaria en el censo, alcanzando al 60\% de la población en Panamá y a casi la mitad de la población en Venezuela (49\%) y Colombia (47\%).5 En América Central los funcionarios siguieron un criterio similar, agrupando a todas las personas de raza mixta en la categoría "ladino" que continuó siendo ampliamente utilizada en la región en los siglos XIX y XX. Solo en Nicaragua los funcionarios distinguieron entre mestizos, mulatos y zambos. ${ }^{6}$

\footnotetext{
3 DE LA SAGRA, Ramón, Historia económico-política y estadística de la isla de Cuba, La Habana, Imprenta de la Viudas de Arazoza y Soler, 1831, pp.1-4; CURTIS Katherine J. y SCARANO, Francisco, "Puerto Rico's Population Padrones, 1779-1802", Latin American Research Review, 2011, 46, 2, pp. 200-213.

4 CAHILL, David, "Colour by Number: Racial and Ethnic Categories in the Viceroyalty of Peru, 1532-1824", Journal of Latin American Studies 1994, 26, 2, p. 339; FISCHER, John, Bourbon Peru, 1750-1824. Liverpool, Liverpool University Press, 2003, p. 55.

5 TOVAR PINZÓN, Hermes et al. Convocatoria al poder del número: Censos y estadísticas de la Nueva Granada, 1750-1830, Bogotá, Archivo General de la Nación, 1994, pp. 68-72; LOMBARDI, John V., People and Places in Colonial Venezuela, Bloomington, Indiana University Press, 1977, p. 132.

${ }^{6}$ Los funcionarios de Costa Rica no usaron el término "ladino" pero agruparon mestizos y mulatos en una sola categoría. Los resúmenes de los recuentos de población de América Central están depositados en el Archivo General de Centro América y me fueron suministrados generosamente por Rina Cáceres.
} 


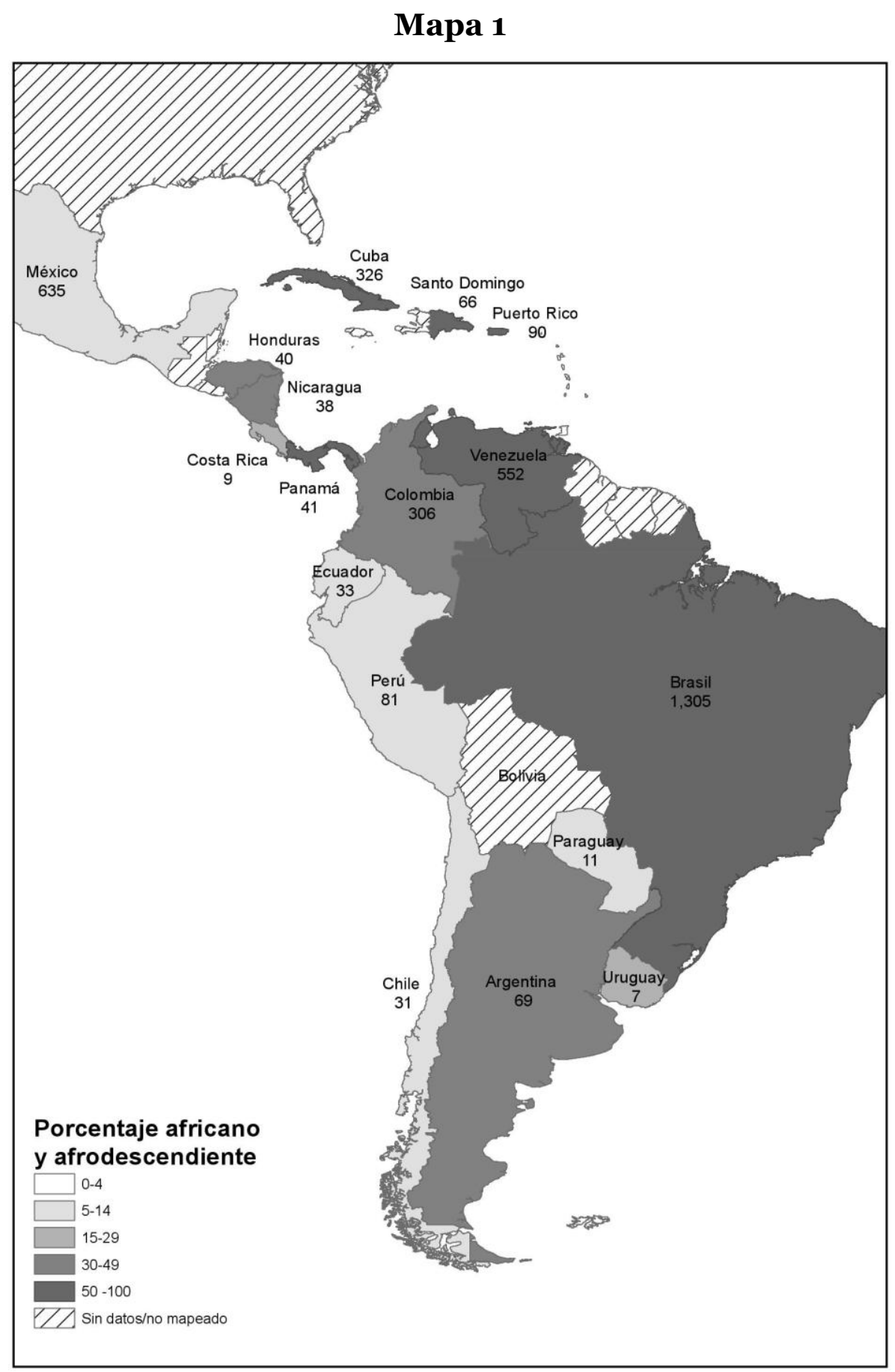

Afro-Latinoamérica, 1800. (Autora: Lena Andrews). Las cifras debajo de los nombres de los países indican el tamaño de la población de africanos y afrodescendientes, en miles.

El recuento de población ordenado en Brasil por la misma época (1776) no incluyó información sobre raza, pero los siguientes censos realizados a comienzos del siglo XIX sí lo hicieron. La mayoría de las capitanías de la colonia (unidades administrativas de nivel provincial) suministraron información sobre los esclavos y las personas de color libres pero no distinguieron entre pretos (negros) y pardos o entre africanos y afro brasileños nativos. Aun en ausencia de 
estas distinciones, estos recuentos de los últimos años del periodo colonial dejan en claro que, por 1800 , Brasil poseía la mayor población africana y afrodescendiente de América Latina, con al menos 1,3 millones de esclavos y pretos y pardos libres. En Hispanoamérica, las mayores poblaciones de negros y pardos estaban en México (635.00o), Venezuela (552.000), Cuba (326.00o) y Colombia (306.00o). Negros y pardos representaban la mayoría de la población en Brasil, Panamá, Santo Domingo, Venezuela, Puerto Rico y Cuba, y grandes minorías (30-50 por ciento) en Colombia, Nicaragua, Argentina y Honduras (ver mapa 1).7

Números tan altos daban cuenta de que las guerras de independencia de las décadas de 1810 y 1820 no pudieron ganarse sin el apoyo político y militar de la población afrodescendiente libre y esclava. Para ganar ese apoyo, los líderes independentistas proclamaron la abolición de las leyes de castas; una vez lograda la independencia los principios de total igualdad civil y el fin de las distinciones de castas fueron incorporados a las constituciones nacionales. En teoría esto debería haber hecho desaparecer las categorías raciales de los censos nacionales. Pero después de la turbulencia y destrucción generalizada que ocasionaron las guerras de independencia, muy pocos gobiernos tenían los recursos y la capacidad para emprender censos de población exhaustivos. En la mayoría de los países de la región, los censos nacionales no comenzaron hasta la segunda mitad del siglo XIX.

Las naciones latinoamericanas realizaron esos censos bajo la sombra del racismo científico que por ese entonces era absolutamente hegemónico en el mundo occidental y eso, claro está, pronosticaba un sombrío futuro para aquellas naciones cuyas poblaciones eran en su mayoría o en una minoría mayor, no blancas. Buscando desmentir esos pronósticos, todas las naciones en la región trataron de blanquear su composición racial atrayendo inmigrantes europeos. Dos de los países que fueron más exitosos en hacerlo, Argentina y Uruguay, no incluyeron la raza en sus censos nacionales pero recolectaron dicha

\footnotetext{
7 Por estas cifras y cómo fueron calculadas, ver ANDREWS, George R., Afro-Latin America, 180o-20oo, Nueva York, Oxford University Press, 2004, 41, pp. 203-207. Además de las fuentes allí citadas, ver, sobre Nicaragua, WOLFE, Justin, The Everyday Nation State: Community and Ethnicity in Nineteenth-Century Nicaragua, Lincoln, University of Nebraska Press, 2007, 7, p. 156; sobre Honduras, EURAQUE, Darío, "La diáspora africana en Honduras: entre la esclavitud colonial y la modernidad del protagonismo Garífuna", en Rina CÁCERES (ed.), Del olvido a la memoria: Africanos y afromestizos en la historia colonial de Centroamérica, San José, Oficina Regional de la UNESCO, 2008, pp. 42-43.
} 
información en los censos municipales de sus ciudades capitales, donde se concentraba la mayoría de los inmigrantes europeos. ${ }^{8}$ No sorprende que los resultados justificaran contundentemente los esfuerzos por blanquear su población realizados por ambos países: en 1887 la población de Buenos Aires era blanca en un 98\%, y la de Montevideo (1884) en un 99\%. Los comentaristas nacionales se basaron en esos datos para proclamar a ambos países como las "repúblicas blancas" de América del Sur, "donde no tardará en quedar su población unificada por completo formando una nueva y hermosa raza blanca producto del contacto de todas las naciones europeas fecundadas el suelo americano". 9

Brasil consiguió atraer unos cuatro millones de inmigrantes entre 1880 y 1930. Sin embargo, dichos inmigrantes tendieron a fluir hacia los estados sureños de San Pablo, Paraná, Santa Catarina y Rio Grande do Sul, evitando las regiones del centro y norte del país. Y muchos de ellos finalmente no permanecieron en Brasil, regresando a sus países de origen o trasladándose a Argentina, Uruguay o Norte América. ${ }^{10}$ Así la composición racial del país continuó siendo fuente de gran preocupación, y los dos primeros censos nacionales, en 1872 y 1890, reunieron información sobre raza. Ellos ofrecieron la auspiciosa noticia de que la población de pretos y pardos sumados había caído de ser la mayoría de la población en 1872 (58\%) a una minoría en 1890 $(47 \%) .{ }^{11}$

De los censos nacionales realizados en América Latina entre 1850 y 1900, aproximadamente la mitad recogieron información sobre raza. ${ }^{12}$ Cuando los países recabaron dicha información, tendieron a hacerlo utilizando las mismas categorías empleadas cien años antes, en los recuentos de población de fines del periodo colonial. Así, mientras en la década de 1880 los primeros censos nacionales de Honduras y Guatemala dividieron la población entre "ladinos" e

\footnotetext{
8 Uruguay contó la raza en el censo de 1852 pero luego dejó de hacerlo en los censos siguientes de 1860 y 1908.

9 La cita pertenece al censo nacional de Argentina de 1895. ANDREWS, George R., The AfroArgentines of Buenos Aires, 180o-190o, Madison, University of Wisconsin Press, 1980, 66, p. 106. Sobre Uruguay, ver ANDREWS, George R., Blacks in the White Nation: A History of AfroUruguay, Chapel Hill, University of North Carolina, 2010, pp. 2-8.

${ }^{10}$ LESSER, Jeffrey, Immigration, Ethnicity, and National Identity in Brazil, 1888 to the Present, New York, Cambridge University Press, 2013, pp. 60-84.

${ }^{11}$ ANDREWS George R., "Racial Inequality in Brazil and the United States: A Statistical Comparison", Journal of Social History, 1992, 26, 2, p. 233.

${ }^{12}$ LOVEMAN, M., ob. cit., p. 113.
} 
indígenas, el censo de Nicaragua de 1883 utilizó las seis categorías del recuento de 1777: indígena, mestizo, mulato, negro, zambo y blanco. Estos diferentes esquemas clasificatorios produjeron resultados radicalmente distintos para la población afrodescendiente: cero por ciento del total nacional en Guatemala y Honduras (donde la población de color se volvió estadísticamente invisible al ser incluida en la categoría "ladino") contra un 43 por ciento en Nicaragua. ${ }^{13}$

Del mismo modo, cuando Colombia reunió información racial en los censos de 1912 y 1918, lo hizo empleando la categoría "mestizo" que era, en lo esencial, la continuación de la categoría "libres de todos los colores" empleada en el recuento de población de 1778. Al incluir mestizos, mulatos, zambos y otro tipo de mezclas raciales, la categoría "mestizo" era la más grande del censo, dando cuenta de la mitad de la población ( $49 \%$ en 1912, 54\% en 1918). Un 9-10 por ciento adicional de la población fue contabilizada como "negro". ${ }^{14}$

La incorporación de la raza en los censos colombianos anticipó una ola de recuentos raciales en la región del Caribe. Nicaragua, Guatemala y Honduras continuaron contando la raza, a los que se sumó Panamá (1911 y 1920), Costa Rica (1927) y El Salvador (1930). El primer censo nacional de República Dominicana (1920) incluyó una pregunta sobre raza, como lo hizo el de México (1920), aunque este último preguntó únicamente sobre población indígena. Cuba (1919 y 1931) continuó recolectando información racial, como lo seguiría haciendo durante todo el siglo XX.

Las décadas de 1910 y 1920 representaron el punto más alto de la marea del racismo científico y de los recuentos raciales en la región. En las décadas de 1920, 1930 y 1940, las críticas de los especialistas sobre la validez científica de la raza, seguidas del completo descrédito que experimentó el concepto por su asociación con las políticas raciales nazis, llevaron a un abandono de los conteos raciales en el período de posguerra. En México, América Central y en los países andinos, los censos continuaron reuniendo información sobre las poblaciones indígenas, pero ahora conceptualizados como grupos definidos en base a parámetros culturales en vez de biológicos. En contraste, los afrodescendientes

13 VALLEJO, Antonio R., Censo general de la República de Honduras levantado el 15 de junio de 1887. Tegucigalpa, Tipografía del Gobierno, 1888; WOLFE, ob. cit, 154-157. Para estimaciones de la población negra y mulata de Honduras (6.7 por ciento en 1910), ver EURAQUE, ob. cit., p. 41.

${ }^{14}$ SMITH, Lynn, "The Racial Composition of the Population of Colombia", Journal of InterAmerican Studies 8, 1996, 2, pp. 214-218. 
fueron vistos como ampliamente integrados a la vida nacional y mucho menos distintos culturalmente. En consecuencia, desaparecieron de los censos de posguerra de casi todos los países, con la excepción de un pequeño grupo: Brasil (1950 y 1960), Costa Rica (1951), Cuba (1953), y República Dominicana (1950 y 1960).15

Rechazando explícitamente el racismo científico de comienzos del siglo $\mathrm{XX}$, en los años de posguerra las naciones latinoamericanas se imaginaron a sí mismas como "democracias raciales" (utilizando el concepto y término brasileño) igualitarias y armoniosas, por lo que no creyeron necesario recoger información censal sobre raza. Si los afrodescendientes ya estaban integrados en las sociedades nacionales en términos de total igualdad ¿cuál sería la explicación, fuera del racismo, de contarlos como un grupo separado? Los movimientos negros de derechos civiles que se formaron en varios países de la región en las décadas de 1970 y 1980 se opusieron vigorosamente a tales argumentos, insistiendo en que la información racial era absolutamente necesaria para determinar si las naciones latinoamericanas habían alcanzado una verdadera igualdad o si persistían las diferencias raciales en el acceso a la salud, la educación, el trabajo, la vivienda y otros beneficios sociales.

La experiencia de Brasil en este sentido sirvió de modelo a los movimientos negros en otros países. La información sobre raza de los censos de 1940 y 1950 había proporcionado evidencia para el análisis sistemático de la desigualdad racial en el país. La decisión del gobierno de no publicar los datos correspondientes a 1960, y luego de eliminar totalmente la pregunta sobre raza en el censo de 1970, hicieron imposible determinar los niveles de desigualdad o, simplemente, el tamaño de la población "preta" y parda durante esas décadas. Cuando el Instituto Brasileiro de Geografia e Estatística (IBGE) se preparaba para realizar el censo de 1980, una coalición de investigadores académicos y activistas afrobrasileños presionó al organismo, logrando que reincorporara la variable raza en los recuentos nacionales. La información proporcionada por los censos de 1980, 1991 y 2000, así como las encuestas nacionales de hogares realizadas anualmente, proporcionaron datos para realizar análisis detallados de las diferencias estadísticas entre la población "preta", parda y blanca. Estos análisis, a su vez, suministraron la mayor parte de la fundamentación para la

15 LOVEMAN, M., ob. cit., pp. 207-249. 
eventual adopción a comienzos de la década del 2000, de políticas nacionales afirmativas en educación y empleo. ${ }^{16}$

Inspirados por lo ocurrido en Brasil, los activistas de Colombia y Uruguay lograron la inclusión de una pregunta sobre raza en el censo nacional (Colombia, 1993 y 2005) o la encuesta nacional de hogares (Uruguay, 1996 y 2006). A comienzos del siglo XXI se dieron otros avances, en gran medida como resultado de la presión ejercida sobre los gobiernos nacionales por agencias internacionales y organizaciones no gubernamentales. Las movilizaciones de las organizaciones de afrodescendientes e indígenas a lo largo de América Latina para protestar contra el 500 aniversario del "descubrimiento" de América por Colón, habían llamado enérgicamente la atención internacional hacia las profundamente enraizadas desigualdades raciales que aquejaban a la región. Al mismo tiempo, agencias de desarrollo tales como el Banco Mundial y el Banco Interamericano de Desarrollo (BID) fueron reconociendo cada vez más que los niveles de extrema desigualdad social no solo eran una lamentable consecuencia del moderno desarrollo capitalista, sino también un importante obstáculo para dicho desarrollo. ${ }^{17}$ Buscando obtener un panorama más completo de esas desigualdades, el Banco Mundial y el BID celebraron reuniones internacionales en 2000 y 2002, reuniendo activistas afrodescendientes e indígenas, investigadores académicos y funcionarios censales para discutir cómo incorporar la información racial a sus recuentos de población. El resultado fue una nueva ronda de censos latinoamericanos en 2010-2011 que, en muchos casos por primera vez en un siglo o más, reunieron datos sobre poblaciones afrodescendientes. ${ }^{18}$

\footnotetext{
${ }^{16}$ HUNT, Mala, "From 'Racial Democracy' to Affrirmative Action: Changing State Policy on Race in Brazil", Latin American Research Review, 2004, 39, 1, pp. 60-89; HERNÁNDEZ, Tanya Katerí, Racial Subordination in Latin America: The Role of the State, Customary Law, and the New Civil Rights Response, New York, Cambridge University Press, 2013, pp. 148-170.

${ }_{17}$ Por evidencias de ese reconocimiento, ver Inter American Development Bank, Facing Up to Inequality in Latin America, Washington, Inter-American Development Bank, 1998: FERRANTI, David et al., Inequality in Latin America: Breaking with History?, Washington, The World Bank, 2004; Gustavo MÁRQUEZ et al. (eds.), Outsiders? The Changing Pattern of Exclusion in Latin America and the Caribbean, Washington, Inter-American Development Bank, 2007.

${ }^{18}$ LENNOX, Corine y MINOTT, Carlos, "Inclusión of Afro Descendents in Ethnic Data Collection: Towards Visibility", International Journal on Minority and Group Rights 2011, 18, 2, pp. 257-275.
} 


\section{Midiendo la Negritud}

Al prepararse para contar a sus ciudadanos de acuerdo a su raza las oficinas censales latinoamericanas enfrentaron el desafío de cómo formular las preguntas relevantes. Esto no era un asunto trivial: al hacer sondeos de las poblaciones indígenas de la región los funcionarios censales habían aprendido que las cifras finales podían variar mucho dependiendo de la pregunta que se formulase. En Panamá, Venezuela y Colombia, el variar las preguntas de los censos tuvo como resultado la duplicación de las cifras de la población indígena entre las décadas de 1980 y los comienzos de la de 1990, seguida por una nueva duplicación (o una cifra mayor aún) a comienzos de la década del 2000. Por ejemplo, en Panamá (2010) la población indígena representó el doce por ciento de la población total, habiendo crecido desde un $5 \%$ en 1980; en Colombia (2005) era 3\% de la población, cuando en 1985 era el 1\%; y en Venezuela (2011), se elevaba al $3 \%$, mientras que era el $1 \%$ en $1981^{19}$.

Colombia tuvo una experiencia similar al contar la población afrodescendiente. En respuesta al lobby de los activistas afro-colombianos, la oficina del censo de Colombia, DANE, accedió a incorporar una pregunta sobre negritud en el censo de 1993, por primera vez desde 1918. Para el enorme disgusto de los activistas, el recuento de 1993 encontró que solo el dos por ciento de la población nacional se identificaba como afro-colombiana. Esta dramática subestimación -los informes gubernamentales preparados en 1970 y 2002 estimaban que los negros y mulatos constituían entre el 20 y el 30 por ciento de la población nacional- fue adjudicada inicialmente a la renuencia de los afro-colombianos para identificarse como tales. Sin embargo, después de un análisis más detenido, la explicación más probable para dicha subestimación se encontró en la forma en que había sido formulada la pregunta en el censo: “ipertenece a alguna etnia, grupo indígena o comunidad negra?”. Con la expresión "comunidad negra" los funcionarios del censo se estaban refiriendo a los asentamientos de población mayoritariamente -o exclusivamente- negra en

19 MARTÍNEZ MAURI, Mónica, "The Social and Political Construction of Racial and Ethnic Categories in National Censuses of Panama, 1911-2010"; LOPERA MESA, Gloria Patricia, "Who Counts Indegenous People, How Are They Counted, and What for? Census Policies and the Construction of Indigeneity in Colombia"; y AGOSTO FERRÁNDEZ, Luis Fernando, "National Censuses and Indigeneity in Venezuela", todos en: Luis Fernando AGOSTO FERRÁNDEZ y Sabine KRADOLFER (eds.), Everlasting Countdowns: Race, Ethnicity and National Censuses in Latin American States, Newcastle upon Tyne, Cambridge Scholars Publishing, 2012. 
las islas de San Andrés y Providencia, el departamento de Chocó en la costa del Pacífico y en otras partes. Los afro-colombianos viviendo fuera de esos asentamientos, en pueblos y barrios con población multirracial -es decir, la gran mayoría de los afro-colombianos- escucharon o leyeron la pregunta, concluyeron que no se refería a ellos y contestaron no, transformando (sin intención alguna) a la población afro-colombiana en estadísticamente invisible. ${ }^{20}$

Decididos a impedir la repetición de esta subestimación, los funcionarios censales diseñaron el censo de población de 2005 enfocado específicamente en los "grupos étnicos" del país: afro-colombianos, indígenas y Rom (gitanos). Todos los individuos no identificados con estos grupos, lo que equivale a decir la mayoría del país blanca y mestiza, fue incluida en la única e indiferenciada categoría "sin pertenencia étnica". Tratando de cubrir todas las posibilidades, la pregunta racial inquirió si "de acuerdo a su cultura, pueblo, o rasgos físicos, es o se reconoce como indígena, Rom" o una de las tres categorías de "negro": isleños de San Andrés /Providencia, residente de la comunidad palenque de San Basilio, o "negro(a)/ mulato(a)/ afrocolombiano(a)/ afrodescendiente". El DANE también financió una campaña publicitaria destinada a sensibilizar al público sobre la pregunta racial y persuadir a los afrocolombianos para que se identificaran como tales. Finalmente, 4,3 millones de colombianos, el 10,5\% de la población total, se identificaron en el censo como negros. Esta cifra estaba muy por debajo de las expectativas de los activistas negros, quienes criticaron al gobierno por no haber incluido la categoría racial de "moreno", muy usada entre los afro-colombianos que viven sobre la costa del Caribe. Aún así, las cifras totales de 2005 fueron mucho más altas que las registradas en 1993. También se correspondían con las de la encuesta realizada en Colombia en 2010 por el Proyecto de Opinión Pública de América Latina (LAPOP), según la cual los autoidentificados como negros y mulatos fueron el 10,4\% de todos los interrogados. ${ }^{21}$

Los cambios introducidos en las preguntas del censo en Ecuador produjeron incrementos menos dramáticos, pero aún así significativos en el

${ }^{20}$ PASCHEL, Tianna, “The Beautiful Faces of My Black People': Race, Ethnicity and the Politics of Colombia's 2005 Census", Ethnic and Racial Studies 36, 10, 2013, p. 1551, 1556n8.

${ }^{21}$ PASCHEL, T., ob. cit., p. 1557; Departamento Administrativo Nacional de Estadísticas (DANE), Colombia, una nación multicultural: Su diversidad étnica, Bogotá, DANE, 2007. Las cifras de LAPOP están disponibles en http://lapop.ccp.ucr.ac.cr/Lapop_English.html. 
tamaño de la población negra de ese país. En 2001 el censo de Ecuador preguntó a los ciudadanos: “¿Ud. cómo se considera?”, ofreciendo las opciones de indígena, "negro (afroecuatoriano)", mulato, mestizo o blanco. En 2010 los funcionarios del censo reformularon la pregunta: “¿Cómo se identifica según su cultura y costumbres?”. También agregaron otra opción, la de "afroecuatoriano(a)/afrodescendiente", que probó ser por lejos la más popular de las categorías referidas a la identidad negra, representando más de la mitad (59\%) de las respuestas relacionadas con ese origen. Como resultado de esa categoría adicional, la población total de negros y mulatos aumentó de un 5\% del total nacional en 2001 al $7 \%$ en 2010. ${ }^{22}$

En Venezuela, en cambio, la atracción de la categoría de "afrodescendiente" fue más limitada. En los dos siglos transcurridos desde la independencia Venezuela nunca había computado la composición racial de su población. En 2008, basándose en las conferencias internacionales realizadas por el BID, el Banco Mundial y las Naciones Unidas, así como las gestiones a nivel nacional realizadas por la Red de Organizaciones Afrovenezolanas, la oficina del censo de Venezuela accedió a incorporar una pregunta sobre raza en el censo de 2011. La pregunta, desarrollada a través de discusiones con organizaciones negras, académicos y funcionarios censales de otros países, preguntó a los venezolanos si, "según sus rasgos físicos, ascendencia familiar, cultura y tradiciones, [usted] se considera "negro(a), afrodescendiente, moreno(a), blanco(a) u 'otro(a)' [o] (ninguna de las anteriores)". Mientras más del $95 \%$ de los venezolanos se identificó como blanco o moreno, el 3\% lo hizo como negro y menos de un $1 \%(0,7)$ como afrodescendiente. ${ }^{23}$

Los relativamente escasos afrodescendientes resultaron ser un grupo muy selecto. Mientras quienes optaron por la categoría "negro" estaban claramente en desventaja en términos educacionales y económicos, en relación con la población blanca, y los morenos ocupaban una posición intermedia entre negros y blancos, los "afrodescendientes" exhibían niveles de logros económicos y educacionales muy semejantes a los del grupo racial blanco. Las tasas de alfabetización y de matrícula universitaria estaban a solo un punto porcentual

22 Ecuador, Censo Nacional de Población y Vivienda, 2010. Disponible en http://redatam.inec.gob.ec/cgibin/RpWebEngine.exe/PortalAction?BASE=CPV2O1O.

23 Venezuela, Instituto Nacional de Estadística (INE), XIV Censo Nacional de Población y Vivienda: Resultados total nacional de la República Bolivariana de Venezuela, Caracas, INE, 2014, p. 29. 
de las tasas de los blancos, y la tasa de pobreza era ligeramente menor entre “afrodescendientes" que entre blancos (tabla 1).

\section{Cuadro 1}

Selección de indicadores socioeconómicos por raza, en porcentajes, Venezuela, 2011

\begin{tabular}{|l|c|c|c|c|}
\hline & Negros & Morenos & Afrodescendientes & Blancos \\
\hline $\begin{array}{l}\text { Porcentaje del total de la } \\
\text { población }\end{array}$ & 2,9 & 51,6 & 0,7 & 43,6 \\
\hline Tasa de pobreza & 37,5 & 30,9 & 21,9 & 22,6 \\
\hline Alfabetismo & 89,9 & 94,7 & 95,8 & 96,5 \\
\hline Matrícula en estudios terciarios & 12,7 & 20,8 & 28,9 & 30,1 \\
\hline
\end{tabular}

Fuente: http://www.redatam.ine.gob.ve/Censo2011/index.html.

La versión del censo de 2011 que está disponible para los investigadores dificulta el análisis detallado de los "afrodescendientes" (o de cualquier otro grupo racial). Podemos manejar la hipótesis de que los "afrodescendientes" son desproporcionadamente urbanos; claramente son más educados que la población negra y morena sumadas. Estos dos factores pueden haberlos hecho más receptivos a los llamados de los activistas negros del país para que se identificaran como (en palabras del censo) "descendientes de africanos $y / o$ africanas que sobrevivieron a la trata negrera, a la esclavitud y forman parte de la diáspora africana en las América y el Caribe y/o es aquella persona que reconoce en sí misma la descendencia africana sobre la base de su percepción, valoración y ponderación de los componentes históricos, generacionales, territoriales, culturales y/o fenotípicos”. ${ }^{24}$

Esta definición, expresando la orientación diaspórica del movimiento negro en Venezuela y otros países, está en marcado contraste con la definición de "negros" dada en el censo: "toda persona de piel fuertemente pigmentada, pelo muy rizado, nariz achatada y labios gruesos. Puede tener prácticas culturales de origen africano, aún cuando no las identifique como tales”. Mientras los "afrodescendientes" estaban definidos en términos de su experiencia histórica colectiva y las elecciones políticas y culturales que habían

\footnotetext{
24 Ibidem., p. 6.
} 
realizado, los "negros" eran definidos por factores físicos y corporales que son innatos y heredados y sobre los que no existe control. ${ }^{25}$

Estos mismos factores eran utilizados para describir a los "morenos", que eran el grupo mayoritario en el censo y la abrumadora mayoría (93\%) de la población no blanca. De acuerdo al censo, los "morenos" son individuos "cuyas características fenotípicas son menos marcadas o pronunciadas que las de la persona definida como negra o negro. Es un término que en algunos contextos puede ser utilizado para suavizar las implicaciones discriminatorias que conlleva ser una persona negra". A lo largo de América Latina, "moreno" juega un rol ambiguo en las identificaciones raciales, ocupando un fluido espacio intermedio entre la negritud y la blancura. Históricamente fue un término asociado con negritud y, en el período colonial, sirvió como un eufemismo cortés para "negro" - un uso que continúa hasta el presente en Venezuela, como sugiere la referencia a "suavizar las implicaciones discriminatorias que conlleva ser una persona negra”. Con el tiempo, ha adquirido un significado asociado con tener piel oscura pero no tan oscura como para ser considerado negro. Una encuesta sobre la composición racial de Venezuela realizada en 2004 por el Laboratorio de Ciencias Sociales, un instituto de investigación privado, agrupó a los morenos y mulatos en una misma categoría racial, representando más de un tercio (36\%) de la población nacional. ${ }^{26}$

Así como se había procedido con los negros y morenos, los blancos fueron definidos en el censo en términos físicos, como "personas cuya tonalidad de piel es clara y que por ello suele ser asociada a poblaciones de origen europeo". Sin embargo, la oficina admitió cierto grado de incertidumbre en esta categoría. "Aunque literalmente implica cuestiones externas como la piel clara, forma y color del cabello y los ojos, entre otras, 'blanco' ha sido usado de distintas maneras en diferentes periodos históricos y lugares. Como

\footnotetext{
25 Ibidem., 66. La definición de "negros" dada por la oficina parece haber sido tomada en forma casi textual de MONTAÑEZ, Ligia, El racismo oculto en una sociedad no racista, Caracas, Editorial Tropykos, 1993, p. 142.

${ }^{26}$ BOLIVAR, Adriana et al., "Discurso y racismo en Venezuela: Un país 'cafré con leche'," en: Teun A VAN DIJK (ed.), Racismo y discurso en América Latina, Barcelona, Editorial Gedisa, 2007, pp. 387-388. Sobre el significado de "moreno" en otros países, ver GOLASH BOZA, Tanya Maria, Yo Soy Negro: Blackness in Peru, Gainesville, University Press of Florida, 2011, 97-101; SUE, Christina A., Land of the Cosmic Race: Race Mixture, Racism, and Blackness in Mexico, New York, Oxford University Press, 2013, pp. 36-45, 55-57; TELLES, Edward, Race in Another America: The Significance of Skin Color in Brazil, Princeton, Princeton University Press, 2004, pp. 82-85.
} 
otras palabras comunes para las etnias humanas, su definición precisa es algo confusa". Una investigación reciente de Edward Telles y René Flores sobre las definiciones de blancura en América Latina encuentra algunas variaciones locales en las mismas pero "una notable consistencia" a través de los países en su aplicación cotidiana. Para la totalidad de la región y para los países grandes, incluido Venezuela, blancura parece tener una práctica y un significado bastante común, al menos en términos de la tonalidad de la piel, y parece así funcionar como una categoría reconocible a nivel transnacional. En Venezuela, casi la mitad de la población, el 44\%, se identificó como perteneciente a esa categoría. ${ }^{27}$

Como Colombia y Ecuador, Costa Rica también modificó sus preguntas censales entre 2000 y 2011. El censo de 2000 preguntó a los censados si "pertenece" a la cultura indígena, negra/afro-costarricense o china. El censo de 2011 abandonó esa formulación cultural y en vez preguntó a los encuestados si se consideraba como negro(a)/ afrodescendiente, mulato(a), chino(a), blanco(a)/mestizo(a), otro(a) o ninguno de los anteriores. Este cambio en la pregunta cuadruplicó el porcentaje de la población que se identificó como afrocostarricense, de un $2 \%$ en 2000 a un $8 \%$ (uno por ciento negro, siete por ciento mulato) en 2011. ${ }^{28}$

En casi todos los países de la región, los activistas y funcionarios censales enfrentaron el problema de determinar qué constituía exactamente la negritud. ¿El censo debía contar solamente a quienes se veían a sí mismos como negros y mulatos (autoidentificación)? ¿O deberían contar a quienes eran considerados por la sociedad como negros y mulatos (identificación externa, quizás por el censista)? Como observa el sociólogo Edgar Telles, "la auto-identificación racial es especialmente útil para entender fenómenos como identidad, disposición para adherir a movimientos sociales, y otros fenómenos sociales que tienden a involucrar la auto-comprensión étnico-racial.... [pero] es menos adecuada para comprender fenómenos sociales como la discriminación, en el que la clasificación está hecha por los otros". Si queremos examinar las dimensiones específicamente raciales de la desigualdad, la discriminación y el prejuicio,

27 INE, XIV Censo Nacional, 29, 66; TELLES, Edward y FLORES, René, "Not Just Color: Whiteness, Nation, and Status in Latin America", Hispanic American Historical Review 93, 3, 2013, pp. 433-434, 447.

${ }^{28}$ LÓPEZ RUIZ, Luis Ángel y DELGADO MONTALDO, David, Situación socioeconómica de la población afrodescendiente de Costa Rica según datos del X Censo Nacional de Población y VI de Vivienda 2011, Ciudad de Panamá, PNUD, 2013, pp. 11-16. 
necesitamos información sobre todos aquellos individuos que la sociedad percibe como no blancos, aún aquellos que puedan no escoger identificarse como tales. "Raza y etnicidad no son solo un tema de identidad o conciencia. También involucran la mirada del otro". 29

Mientras tanto ¿qué pasa con aquellas personas que no muestran signos visibles de ascendencia africana pero que sin embargo reconocen, o afirman, tener algún grado de herencia africana? Los movimientos afro en Argentina y Uruguay exigieron a sus gobiernos incluir a este tipo de individuos en los censos nacionales, al menos en parte para aumentar el tamaño de la población afrodescendiente. En respuesta a estas demandas de los movimientos, el censo argentino de 2010 inquirió a los encuestados, en una sola pregunta, si se consideraban afrodescendientes o tenían algún antepasado que fuera afrodescendiente o "de origen africano", remontándose hasta sus bisabuelos. Aun con una pregunta tan amplia, solo el $0,4 \%$ de la población argentina respondió afirmativamente. 30

Después de experimentar (en la encuesta nacional de hogares de 1996) con la identificación racial, Uruguay también optó por la ascendencia, sin especificar cuán atrás en su historia familiar deberían ir los encuestados. Mientras la encuesta de 1996 preguntó “¿a qué raza Ud. cree pertenecer?”, la encuesta de hogares de 2006, y luego el censo de 2011, cambiaron la pregunta a “cree tener ascendencia...?” y permitieron a los encuestados registrarse en más de una categoría. Como reconocieron los funcionarios censales, las preguntas de 1996 y 2006/2011 no eran equivalentes y por lo tanto no midieron lo mismo. "En 2006 [y 2011] el término central incorporado en la pregunta fue la ascendencia. Este concepto remite a la herencia genética de las personas, pero no involucra necesariamente su aspecto físico". Este cambio aumentó la población afro-uruguaya (o quizás, mejor dicho, la población que afirma tener algún grado de ascendencia africana) de 6\% en 1996 a 9\% en 2006 y 8\% en 2011.31

29 TELLES y PERLA Edward E., Pigmentocracies: Ethnicity, Race and Color in Latin America, Chapel Hill, University of North Carolina Press, 2014, p. 10.

${ }^{30}$ Instituto Nacional de Estadística y Censos (INDEC), Censo nacional de población, hogares y viviendas 2010, vol. 1, Buenos Aires, INDEC, 2012, pp. 295-305.

${ }^{31}$ BUCHELI, Marisa y CABELLA, Wanda, Perfil demográfico y socioeconómico de la población uruguaya según su ascendencia racial, Montevideo, INE, 2008, 10-11. Ver también CABELLA, Wanda et al., La población afrouruguaya en el Censo 2011, Montevideo, Ediciones Trilce, 2013. 
Mientras tanto, las dos naciones latinoamericanas que han reunido consistentemente información sobre negritud a lo largo de todo el siglo XX, Brasil y Cuba, continúan haciéndolo en la década de 2000, utilizando su tradicional sistema de categorías basadas en el color. Los censos de Cuba de 2002 y 2012 pidieron a los encuestados que se categorizaran de acuerdo al "color de la piel", y el censo brasileño preguntó a los individuos por su "color o raza”. Los afro-cubanos constituían el 36\% del total nacional en 2012, en relación con el 35\% en 2002.32 El censo de Brasil de 2010 mostró a los afrobrasileños como una ajustada mayoría de la población nacional, con el 51\%. Esto representó un incremento considerable en relación con el año 2000, cuando los pretos y pardos brasileños habían ascendido al 45\% de la población nacional total. Parte de ese aumento se origina sin duda en la creciente disposición de los afro-brasileños para reconocer, o aún abrazar, la ascendencia africana y el status de no blancos. Otra parte del mismo, sin embargo, se debe a factores demográficos, en particular a las tasas de natalidad que se mantienen más altas entre las mujeres afro-brasileñas que entre las mujeres blancas. Las tasas de fertilidad cayeron drásticamente en Brasil para ambos grupos raciales durante los últimos treinta años, y en el caso de las mujeres blancas cayeron por debajo del nivel de reemplazo de 2,1 hijos por mujer (número que permite que la población permanezca estable, sin crecimiento ni disminución). En el caso de las mujeres afrodescendientes los nacimientos apenas superan el nivel de reemplazo, llevando a un crecimiento más rápido de la población preta y parda que de la población blanca.33

La comparación de las diferencias raciales en las tasas de fertilidad es precisamente uno de los ejemplos del tipo de análisis de diferencias raciales y desigualdades que la ronda de censos nacionales de 2010 permite realizar. Podríamos ahondar mucho más en el análisis de esta información, y espero que al menos algunos de los lectores se sientan animados a hacerlo. Todos los censos están disponibles en los sitios web de las oficinas nacionales encargadas de realizarlos o en el sitio web de la Comisión Económica para América Latina y el

${ }^{32}$ Oficina Nacional de Estadística e Información (ONEI), Resumen adelantado: Resultados definitivos de indicadores seleccionados en Cuba, provincias y municipios, La Habana, ONEI, n.d., pp. 17-18, 68-74.

33 PAIXAO, Marcelo et al. (eds.) Relatório anual das desigualdades raciais no Brasil, 2009201O, Rio de Janeiro, Editora Garamond, 2010, pp. 81-83. 
Caribe de las Naciones Unidas.34 Estas bases de datos permiten descomponer los grupos raciales en subgrupos por género, edad, región u otras variables, o, alternativamente, investigar los aspectos raciales de dichas variables (género, raza, etc.). Esto abre enormes oportunidades para futuras investigaciones y análisis de la desigualdad racial en la región, oportunidades que seguirán ampliándose a medida que se realicen futuros censos y los mismos sean puestos a disposición del público.

Mientras tanto, podemos utilizar las cifras de los censos de 2010 (o, en el caso de aquellos países que no contaron la raza en 2010, las de la encuesta de LAPOP de 2010) para construir un amplio panorama de población negra de la región (mapa 2). En ese año, la población afrodescendiente de América Latina era estimada en unos 131 millones de personas, representando el 24\% del total regional. La mayoría de los afrodescendientes (97 millones) vivían en Brasil, con otros 15 millones en Venezuela y 8 millones en la República Dominicana. En estos tres países la mayoría de la población era afrodescendiente; en Cuba más de un tercio de la población era afrodescendiente. En el resto de los países había un $12 \%$ de afrodescendientes o menos. 35

34

http://celade.cepal.org/redbin/RpWebEngine.exe/Portal?\&MODE=BASE\&ITEM=CPVAMLAT. 35 Todas las cifras corresponden a la ronda de censos nacionales de 2010, a excepción de Chile, República Dominicana, Honduras, México, Nicaragua, Paraguay y Perú, que no recogieron información sobre sus poblaciones afrodescendientes. Para estos países utilicé los porcentajes de individuos que se identificaban como negros y mulatos en la encuesta LAPOP 2010 y apliqué esos porcentajes a los resultados totales de los censos de 2010 para generar una estimación de la población afrodescendiente. Los datos de LAPOP están disponibles en http://lapop.ccp.ucr.ac.cr/Lapop_English.html. Por cifras comparables para la región basadas en un procedimientos estadístico similar, ver TELLES et al., Pigmentocracies, pp. 26-27. 


\section{Mapa 2}

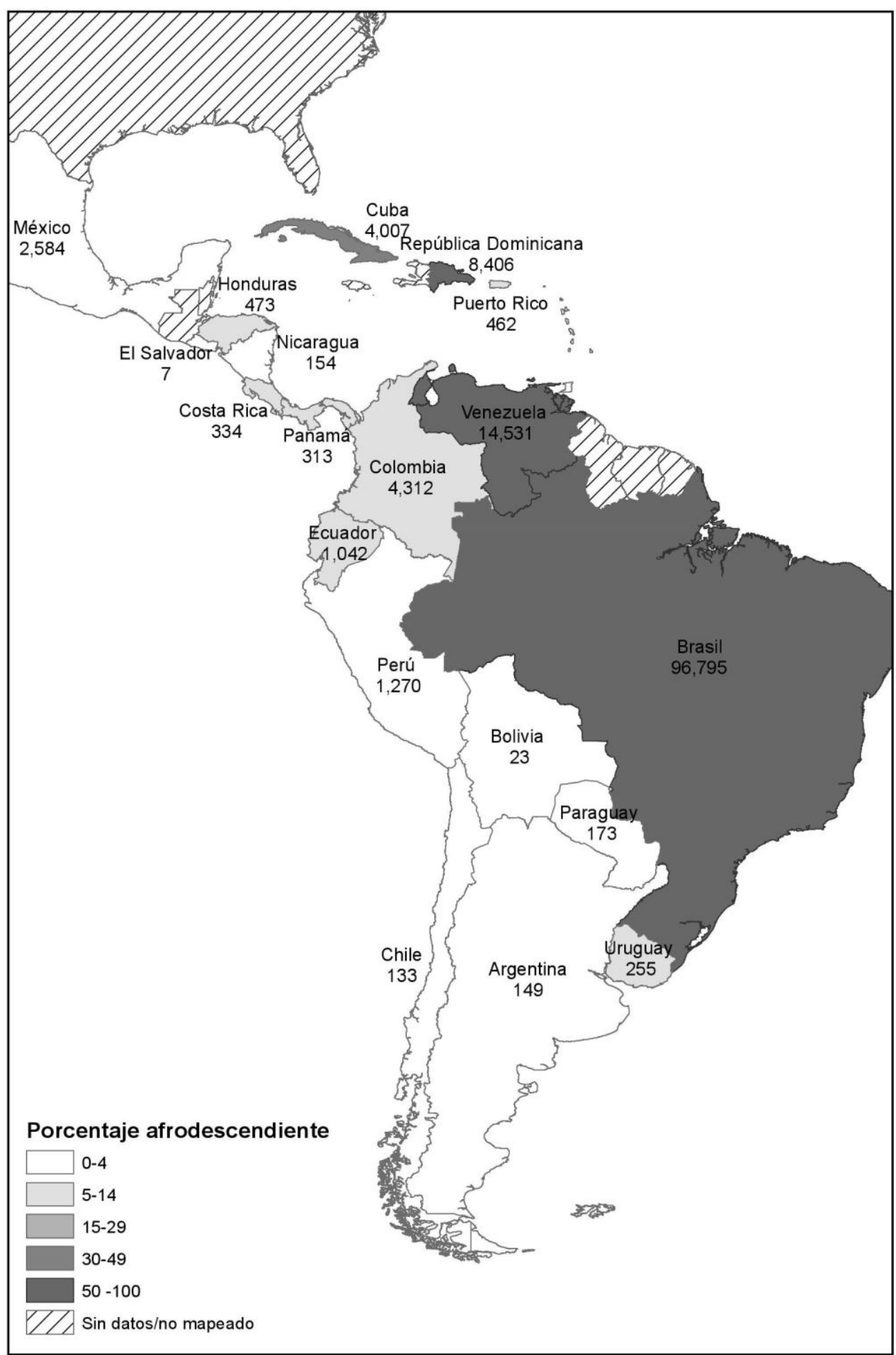

Afro-Latinoamérica, 2010. Autora: Lena Andrews. Las cifras debajo de los nombres de los países indican el tamaño de la población afrodescendiente, en miles.

Hemos recorrido un largo camino desde aquellos primeros recuentos de población de fines del siglo XVIII y quizás un camino aún más largo desde los censos de los últimos cincuenta años, la mayoría de los cuales no proporcionaba 
ninguna información sobre los significados y las dinámicas demográfico-raciales en la región. Ahora tenemos acceso a abundante información de este tipo, con la promesa de que habrá más; los afro-latinoamericanos ya no son invisibles estadísticamente.

\section{Bibliografía y Fuentes}

\section{Bibliografía}

AGOSTO FERRÁNDEZ, Luis Fernando, "National Censuses and Indigeneity in Venezuela" en AGOSTO FERRÁNDEZ Luis Fernando y KRADOLFER, Sabine (eds.), Everlasting Countdowns: Race, Ethnicity and National Censuses in Latin American States, Newcastle upon Tyne, Cambridge Scholars Publishing, 2012.

ANDREWS, George R.

-Afro-Latin America, 180o-20oo, Nueva York, Oxford University Press, 2004.

-The Afro-Argentines of Buenos Aires, 180o-19oo, Madison, University of Wisconsin Press, 1980, 66.

-Blacks in the White Nation: A History of Afro-Uruguay, Chapel Hill, University of North Carolina, 2010.

-"Racial Inequality in Brazil and the United States: A Statistical Comparison", Journal of Social History, 1992, 26, 2.

BOLIVAR, Adriana et al., "Discurso y racismo en Venezuela: Un país 'cafré con leche'," en Teun A VAN DIJK,. (ed.), Racismo y discurso en América Latina, Barcelona, Editorial Gedisa, 2007.

BUCHELI, Marisa y CABELLA, Wanda, Perfil demográfico y socioeconómico de la población uruguaya según su ascendencia racial, Montevideo, INE, 2008.

CABELLA, Wanda et al., La población afrouruguaya en el Censo 2011, Montevideo, Ediciones Trilce, 2013.

CAHILL, David, "Colour by Number: Racial and Ethnic Categories in the Viceroyalty of Peru, 1532-1824”, Journal of Latin American Studies 1994, 26, 2.

CURTIS Katherine J. y SCARANO, Francisco, "Puerto Rico's Population Padrones, 1779-1802”, Latin American Research Review, 2011, 46, 2. 
EURAQUE, Darío, "La diáspora africana en Honduras: entre la esclavitud colonial y la modernidad del protagonismo Garífuna”, en Rina CÁCERES, (ed.), Del olvido a la memoria: Africanos y afromestizos en la historia colonial de Centroamérica, San José, Oficina Regional de la UNESCO, 2008.

FERRANTI, David et al., Inequality in Latin America: Breaking with History?, Washington, The World Bank, 2004.

FISCHER, John, Bourbon Peru, 1750-1824. Liverpool, Liverpool University Press, 2003.

HERNÁNDEZ, Tanya Katerí, Racial Subordination in Latin America: The Role of the State, Customary Law, and the New Civil Rights Response, New York, Cambridge University Press, 2013.

GOLASH BOZA, Tanya Maria, Yo Soy Negro: Blackness in Peru, Gainesville, University Press of Florida, 2011.

HUNT, Mala, “From 'Racial Democracy' to Affrirmative Action: Changing State Policy on Race in Brazil”, Latin American Research Review, 2004, 39, 1.

INTER AMERICAN DEVELOPMENT BANK, Facing Up to Inequality in Latin America, Washington, Inter-American Development Bank, 1998.

LENNOX, Corine y MINOTT, Carlos, "Inclusión of Afro Descendents in Ethnic Data Collection: Towards Visibility", International Journal on Minority and Group Rights 2011, 18, 2.

LESSER, Jeffrey, Immigration, Ethnicity, and National Identity in Brazil, 1888 to the Present, New York, Cambridge University Press, 2013.

LOMBARDI, John V., People and Places in Colonial Venezuela, Bloomington, Indiana University Press, 1977.

LOPERA MESA, Gloria Patricia, "Who Counts Indegenous People, How Are They Counted, and What for? Census Policies and the Construction of Indigeneity in Colombia" en Luis Fernando AGOSTO FERRÁNDEZ y Sabine KRADOLFER (eds.), Everlasting Countdowns: Race, Ethnicity and National Censuses in Latin American States, Newcastle upon Tyne, Cambridge Scholars Publishing, 2012.

LÓPEZ RUIZ, Luis Ángel y DELGADO MONTALDO, David, Situación socioeconómica de la población afrodescendiente de Costa Rica según datos del X Censo Nacional de Población y VI de Vivienda 2011, Ciudad de Panamá, PNUD, 2013.

LOVEMAN, Mara, National Colors: Racial Classification and the State in Latin America, New York, Oxford University Press, 2014. 
MARTÍNEZ MAURI, Mónica, "The Social and Political Construction of Racial and Ethnic Categories in National Censuses of Panama, 1911-2010”.

MÁRQUEZ, Gustavo et al., eds., Outsiders? The Changing Pattern of Exclusion in Latin America and the Caribbean, Washington, Inter-American Development Bank, 2007.

MONTAÑEZ, Ligia, El racismo oculto en una sociedad no racista, Caracas, Editorial Tropykos, 1993.

PAIXAO, Marcelo et al. (eds.) Relatório anual das desigualdades raciais no Brasil, 2009-201O, Rio de Janeiro, Editora Garamond, 2010.

PASCHEL, Tianna, “The Beautiful Faces of My Black People’: Race, Ethnicity and the Politics of Colombia's 2005 Census”, Ethnic and Racial Studies $36,10,2013$.

SMITH, Lynn, "The Racial Composition of the Population of Colombia”, Journal of Inter-American Studies 8, 1996, 2.

SUE, Christina A., Land of the Cosmic Race: Race Mixture, Racism, and Blackness in Mexico, New York, Oxford University Press, 2013.

TELLES, Edward, Race in Another America: The Significance of Skin Color in Brazil, Princeton, Princeton University Press, 2004.

TELLES, Edward y FLORES, René, "Not Just Color: Whiteness, Nation, and Status in Latin America”, Hispanic American Historical Review 93, 3, 2013 .

TELLES y PERLA Edward E., Pigmentocracies: Ethnicity, Race and Color in Latin America, Chapel Hill, University of North Carolina Press, 2014.

TOVAR PINZÓN, Hermes et al. Convocatoria al poder del número: Censos y estadísticas de la Nueva Granada, 1750-1830, Bogotá, Archivo General de la Nación, 1994.

WOLFE, Justin, The Everyday Nation State: Community and Ethnicity in Nineteenth-Century Nicaragua, Lincoln, University of Nebraska Press, 2007, 7 .

\section{Fuentes}

celade.cepal.org/redbin/RpWebEngine.exe/Portal?\&MODE=BASE\&ITEM=CP VAMLAT.

DE LA SAGRA, Ramón, Historia económico-política y estadística de la isla de Cuba, La Habana, Imprenta de la Viudas de Arazoza y Soler, 1831. 
DEPARTAMENTO ADMINISTRATIVO NACIONAL DE ESTADÍSTICAS (DANE), Colombia, una nación multicultural: Su diversidad étnica, Bogotá, DANE, 2007.

INSTITUTO NACIONAL DE ESTADÍSTICA (INE), XIV Censo Nacional de Población y Vivienda: Resultados total nacional de la República Bolivariana de Venezuela, Caracas, INE, 2014.

Instituto Nacional de Estadística y Censos (INDEC), Censo nacional de población, hogares y viviendas 2010, vol. 1, Buenos Aires, INDEC, 2012.

ECUADOR, Censo Nacional de Población y Vivienda, 2010. Disponible en http://redatam.inec.gob.ec/cgibin/RpWebEngine.exe/PortalAction?BAS $\mathrm{E}=\mathrm{CPV} 2010$.

lapop.ccp.ucr.ac.cr/Lapop_English.html.

OFICINA NACIONAL DE ESTADÍSTICA E INFORMACIÓN (ONEI), Resumen adelantado: Resultados definitivos de indicadores seleccionados en Cuba, provincias y municipios, La Habana, ONEI, n.d.

VALLEJO, Antonio R., Censo general de la República de Honduras levantado el 15 de junio de 1887, Tegucigalpa, Tipografía del Gobierno, 1888. 